\title{
Assessing Quality of Life in ESRD Patients: Sleep Quality and Associated Factors
}

\author{
1st. Made Suandika \\ Lecture, School of Nursing \\ Harapan Bangsa University \\ Central Java, Indonesia
}

\author{
2nd. Woung Ru Tang \\ Professor, school of Nursing \\ College of Medicine \\ Chang Gung University, ROC, Taiwan
}

\begin{abstract}
Background: Sleep disturbance is the most common problem in patients with end-stage kidney disease (ESRD). This study investigated the adverse events of sleep quality that affect Quality of Life (QoL) and identified risk factors of sleep disorders with general participant's characteristics, related to laboratory data on ESRD patients undergoing hemodialysis in Central Java, Indonesia.

Methods: This research was a cross-sectional study involving 123 participants. The research instruments used were a personal information questionnaire, the Pittsburgh Sleep Quality Index (PSQI) questionnaire, and the WHOQOL-BREF questionnaire which aimed to assess sleep quality affecting quality of life and related factors in ESRD patients. The questionnaires were translated into Indonesian and completed by the participants during the hemodialysis session.

Results: Most participants with PSQI> 5 (poor sleep quality) were female $(55.05 \%)$ with a long history of dialysis> 7 years $(38.53 \%)$. The PSQI score has an inverse relationship with age, marital status, alcohol intake and smoking history. The results show that multivariate logistic regression analysis with dialysis vintage with $p$ value $(0.005)$ was a more significant predictor than $p$ value of BMI $(0.002)$ and $p$ value of cholesterol level $(0.001)$. There was a significant predictor of poor sleep quality.

Conclusion: The research showed that the majority of the participants were at productive age levels ranging from 3650 years $(\mathbf{5 1 . 3 8 \%})$, and female generally complained of sleep disturbances in ESRD patients.
\end{abstract}

Keywords: hemodialysis, sleep disorder, cholesterol, BMI, dialysis vintage

\section{INTRODUCTION (HEADING l)}

the world's most serious health problem is end stage renal disease (ESRD). the incidence of ESRD increased $8 \%$ five years ago. [1]. although many epidemiological studies report an increase in ESRD undergoing replacement therapy worldwide, the size of the problem may not be fully interpreted. patients with ESRD experience discomfort in the skin such as itching which causes poor sleep complaints. sleep quality is considered as a potential predictor of death and it reduces the quality of life of these patients. [2]

increasing the quality of dialysis is no longer the main goal of patients with ESRD because dialysis techniques and medical care can reduce birth and death rates. [3] however, it has been reported that the prevalence of health problems associated with quality of life in patients with ESRD is higher.[4] [5] line with previous studies, $50-80 \%$ of patients with ESRD experience sleep disorders, such as sleeping difficulty and falling asleep, waking up too early, daytime sleepiness, and leg trembling. [1] [6] in shuci anand et al's [7] study, the incidence of sleep disorders among ESRD patients with the appearance of the metabolic syndrome, diabetes, and risk factors for bound hypertension was related to the problems of obesity and nutrition transition. poor sleep deprivation can greatly affect mood, energy, mental acuity, and decreased_of stress. in addition, long-term chronic sleep loss can make adverse effects on mental and physical health.

Good quality of sleep is basically needed by all people as it can provide a break of mental and physiological periods. during sleep, the sympathetic tone decreases and the parasympathetic tone increases, causing a decrease of heart rate, arterial blood pressure, and cardiac output. therefore, individuals need to be aware of the need for sleep at night to get a healthy sleep and good quality of life. in addition, several studies have shown that sleep disorders alter the innate functional cellular immune response. [8] it is estimated that the incidence of chronic kidney failure is around 40-60 cases per million population per year in developing countries. this also happens in indonesia where there are 100 patients with chronic kidney failure per million population or around 20,000 cases per year ([9] in addition, the prevalence of hemodialysis in central java indonesia is high from year to year. about $50 \%$ of patients with chronic ESRD undergoing hemodialysis (hd) experience insomnia and other sleep disorders. [10] therefore, this study explores the sleep quality of patients with ESRD who live in central java. it also evaluates possible related factors, whether or not it has an impact on quality of life (qol) and identifies risk factors in patients with ESRD who undergo hemodialysis. ease of use

\section{METHODOLOGY}

Study design--- This cross-sectional study was conducted from August to December 2016. The instrument used in this study was Pittsburgh Sleep Quality Index (PSQI) which was translated into Indonesian. The Cronbach 
alpha is around 0.79 and the content validity score is 0.89 . Construct validity showed a correlation between components and global PSQI scores, and group validity is known to be significant ( $\mathrm{p}<0.001)$. Sensitivity is one and specificity is 0.81 with cut off 5 . This instrument is proven to be valid and reliable. [11] Sleep quality and laboratory data including demographic data and completing the questionnaire were conducted at the same time. [12].

Setting and samples----There were 126 patients undergoing regular hemodialysis at two different dialysis centers in the Regional General Hospital such as Banyumas Hospital and Prof. Dr.Margono Soekardjo Hospital of Central Java in which those hospitals have high prevalence of dialysis patient every years [7]. All of the patients involved in the study were the participants. They filled a questionnaire about sleep quality and quality of life. The participants were over 18 years old and had received hemodialysis therapy for more than three months to $>7$ years. Patients who experienced severe heart failure, respiratory problems (severe chronic obstructive pulmonary disease), and psychological problems or patients who were hospitalized one month before were excluded from this study. In addition, three patients were excluded from this study because of incomplete data.

\section{Ethical considerations--- Most importantly,}

the participants must answer the questionnaire and be able to communicate with the researchers. In the end, the complete data from 123 patients were collected for statistical analysis. The purpose of this study was to explain completely to all patients, and informed consent was obtained from all participants. This study was approved by the research committee of Jenderal Soedirman University, Purwokerto, Indonesia through an ethics approval number: 158 / KEPK / VII / 2016.

Measurement and data collection/procedure---Sleep Quality Assessment---The questionnaire used in this study was adopted from the Pittsburgh Sleep Quality Index (PSQI). It was employed to assess the dimensions of sleep in one month. PSQI consists of 19 questions covering seven components which are subjective sleep quality, latency, efficiency, duration, disturbances during sleep, use of sedative drugs, and sleep disturbances during the day. The global score in this questionnaire is between 0 and 21 . A higher score indicates how poor the sleep quality of the participants is. A score $>5$ or higher indicates that the person has a sleep problem.[11] [6] This questionnaire has been used in many studies to measure sleep quality and determine sleep disorders. The questionnaire has very good validity and reliability. The results of the study defined PSQI> 5 as a standard for analyzing poor sleep quality.

Quality of Life Assessment----The QOL questionnaire was used to assess the quality of life as determined by the general instruments of the World Health Organization (WHOQOL-BREF). The WHOQOL-BREF questionnaire has been translated into 19 different languages, including Indonesian. The Indonesian version of WHOQOL-BREF has been validated and has been proven to have good content validity, discriminatory validity, reliability retest, and internal consistency. [13]. The Indonesian translated QoL questionnaire (WHOQOL-BREF) consisted of 26 items

including four domains i) physical condition consisting of 7 questions, ii) psychological condition consisting of 6 questions, iii) social relations comprising 3 questions and iv) environment consisting of 8 questions. Each question is given a value of 1 to 5 , and a higher value indicates a better quality of life. The score of the domain is calculated by multiplying the average of each aspect by 4 . The domain is not given a score if $\geq 20 \%$ of the questions are not answered by the respondent. [13] domain score to a scale of $0-100$. A higher score reflects a better quality of life. By comparing the scores for testing, QOL assessments were carried out using the Statistical Package for Social Sciences (SPSS) 22.0 software.

Demographic and Laboratory Data----In this study, demographic and laboratory data were collected with the assistance of nursing staffs. Demographic data covered information required in this study, which includes gender, marital status, alcohol intake, smoking, dialysis vintage (years), working status, BMI status, and age. Meanwhile, the laboratory data were collected to obtain information about biochemistry test, such as Anti-HCV, HB, Hct, BUN, Urea blood level, Creatinine blood level, albumin, Calcium, Kalium, Natrium, Triglyceride, Cholesterol which were provided by the monthly routine examination at the dialysis centers. In addition, medical documentations and medicine records as medical profile of those patients present required information such as dialysis information, marital status, alcohol intake, smoking, working status.

Data Analysis ----- Patient's data were collected and the signing of information was required by the ethics committee. The data are a continuous variable presented in the form of \pm 1 standard deviation (SD) in the normal distribution with frequency and percentage. Differences in variables such as demographic factors and medical profiles between good and bad sleep and also good and bad QOL were tested by the chisquare test ( $\chi 2$ test) and Fisher Exact test. The Spearman relationship test was used to test normal non-distributed variables. Significant risk factors for poor sleep quality and poor QOL were analyzed by stepwise logistic regression. All analyzes were carried out using SPSS version 22 (SPSS Inc., Chicago, IL, USA). A P value of $<0.05$ was considered statistically significant.

\section{FINDINGS AND DISCUSSION}

There were 123 patients with ESRD involved in this study (see table 1). All of them completed the questionnaire. The results of the Spearman correlation analysis show that there is a relationship between gender and sleep quality. From the participants, $44.95 \%$ were male and $55.05 \%$ were female. Patients with poor sleep quality (PSQI $>5$ ) comprised more than half $(88.6 \%)$ of the participants and Poor Quality of Life (QoL) was found (49.6\%). The following table presents the characteristics of the social-demographic and 
para-clinical laboratory variables_(see table 1), and about the multiple regression we can see on table 2

\section{Table 1 Demography characteristic}

\begin{tabular}{|c|c|c|c|c|c|}
\hline \multirow{3}{*}{ Variables } & Gooc & & & & \multirow{3}{*}{$\mathrm{p}$} \\
\hline & \multicolumn{2}{|c|}{$n=14$} & \multicolumn{2}{|c|}{$>5 n=109$} & \\
\hline & $F$ & $\%$ & $E$ & $\%$ & \\
\hline Age (years) & & & & & 0.464 \\
\hline $20-35$ & 6 & $42.86 \%$ & 33 & $30.28 \%$ & \\
\hline $36-50$ & 7 & $50.00 \%$ & 56 & $51.38 \%$ & \\
\hline $51-65$ & 1 & $7.14 \%$ & 20 & $18.35 \%$ & \\
\hline Gender (M/F) & & & & & 0.018 \\
\hline Male & 11 & $78.57 \%$ & 49 & $44.95 \%$ & \\
\hline Female & 3 & $21.43 \%$ & 60 & $55.05 \%$ & \\
\hline Marcital status & & & & & 0.600 \\
\hline Unmarricd & 2 & $14.29 \%$ & 22 & $20.18 \%$ & \\
\hline Married & 12 & $85.71 \%$ & 87 & $79.82 \%$ & \\
\hline Alcohol Intake & & & & & 0.215 \\
\hline Jinalcohel & 13 & $92.86 \%$ & 86 & $78.90 \%$ & \\
\hline Alcoholic & 1 & $7.14 \%$ & 23 & $21.10 \%$ & \\
\hline Smoking & & & & & 0.600 \\
\hline Non-smoker & 2 & $14.29 \%$ & 22 & $20.18 \%$ & \\
\hline Current Smoker & 12 & $85.71 \%$ & 87 & $79.82 \%$ & \\
\hline \multicolumn{3}{|c|}{ Dialysis Vintage (years) } & & & 0.043 \\
\hline$<1$ year & 6 & $42.86 \%$ & 18 & $16.51 \%$ & \\
\hline $1-3$ year & 4 & $28.57 \%$ & 24 & $22.02 \%$ & \\
\hline $4-7$ years & 3 & $21.43 \%$ & 25 & $22.94 \%$ & \\
\hline$>7$ year & 1 & $7.14 \%$ & 42 & $38.53 \%$ & \\
\hline Working Status & & & & & 0.350 \\
\hline Not working & 12 & $85.71 \%$ & 81 & $74.31 \%$ & \\
\hline Working & 2 & $14.29 \%$ & 28 & $25.69 \%$ & \\
\hline $\mathrm{BMI}\left(\mathrm{Kg} / \mathrm{M}^{2}\right)$ & & & & & 0.002 \\
\hline Normal: & 14 & $100.00 \%$ & 52 & $47.71 \%$ & \\
\hline Overweight & 0 & $0.00 \%$ & 54 & $49.54 \%$ & \\
\hline Moderately obes & 0 & $0.00 \%$ & 2 & $1.83 \%$ & \\
\hline Severely obese & 0 & $0.00 \%$ & 1 & $0.92 \%$ & \\
\hline
\end{tabular}

By $x^{2}$ test, $P<0.05$, and spearmen correlation

Table 2. Stepwise logistic regression analysis

\begin{tabular}{lcccccc}
\hline Variables & \multicolumn{3}{c}{ Step 1 } & \multicolumn{5}{c}{ Step 7 } \\
\cline { 2 - 7 } & OR & $95 \% \mathrm{Cl}$ & p Valuc & OR & $95 \% \mathrm{Cl}$ & p Value \\
\hline Gender & 3.164 & $0.704-14.218$ & 0.133 & & & \\
Dialysis, vintage & 2.119 & $1.157-3.88$ & 0.015 & 2.335 & $1.285-4.242$ & $0.005 * *$ \\
BMI & 1.877 & $1.236-2.849$ & 0.003 & 1.864 & $1.264-2.749$ & $0.002 * *$ \\
\hline BUN & 1.098 & $0.969-1.245$ & 0.144 & & & \\
Albumin & 0.328 & $0.071-1.505$ & 0.151 & & & \\
Kalium & 0.98 & $0.776-1.237$ & 0.862 & & & \\
Anti_HCX & 0.082 & $0.007-0.95$ & 0.045 & & & \\
Triglyceride & 1.015 & $0.998-1.032$ & 0.079 & & & \\
Cholesterol & 1.032 & $1.012-1.053$ & 0.002 & 1.034 & $1.014-1.055$ & $0.001 * *$ \\
\hline
\end{tabular}

Most participants with PSQI> 5 (poor sleep quality) were women $(55.05 \%)$ with a long history of dialysis> 7 years $(38.53 \%)$. PSQI scores have an inverse relationship with age, marital status, alcohol intake and smoking history. The results showed that multivariate logistic regression analysis with vintage $p$ value $(0.005)$ of dialysis was a more significant predictor than $\mathrm{p}$ Value of BMI (0.002) and cholesterol level p Value (0.001). There was a significant predictor of poor sleep quality (see table 1 and table 2).

Several studies have shown a significant relationship between serum cholesterol levels and sleep quality or triglyceride levels and quality of life. [14] In this study, we also found that patients with higher serum cholesterol levels had worse sleep quality. Sabet et al mentioned that the risk of poor sleep quality was found to be higher in patients with a longer history of dialysis $>1$ year. [15].

Poor sleep quality in this study is due to conditions experienced by hemodialysis patients who have difficulty sleeping due to very high cholesterol levels in the blood, which includes quantitative aspects of sleep, such as sleep duration, sleep latency, frequency of awakening, and subjective aspects from deep sleep and sleep. Poor sleep quality is often associated with general health problems, which are also bad. [16] In this study, participants with PSQI> 5 (poor sleep quality) were women (51.02\%). Sleep disorders can cause problems related to the endocrine, immune, nervous and cardiovascular systems; one of them is hypertension. According to Bailie B et al., short sleep time is associated with a greater risk of cardiovascular disease.[1] Incidence or prevalence of high triglycerides or low HDL cholesterol levels has been found among individuals who sleep too little, especially in women. [17] One study also illustrates that shorter sleep duration produces higher BMI, total cholesterol levels and triglycerides (Bjovantn B et al 2007). In this study, the actual hours of sleep were recorded in the PSQI questionnaire.

High cholesterol levels can also cause several complications, including stroke, myocardial infarction, encephalopathy, and kidney failure. This gender research is a biological factor that distinguishes between men and women; gender also distinguishes the quality of sleep between the two. Some studies also mentioned that sleep disorders in men are better than women. Any disease can basically attack humans, both men and women, but there are some symptoms that have different frequencies between men and women in hemodialysis patients; one of which is sleep disorders.

This study shows that demographic factors, lifestyle factors, biological factors and dialysis factors are not related to sleep quality with $\mathrm{p}$ value $>0.05$. Demographic factors consist of age, sex and occupation. Age can increase and decrease susceptibility to certain diseases. Age is one of the risk factors for chronic kidney failure over time. Kidney function will decrease so that the kidneys are unable to do their job. Changes and stress in old age can be caused by anxiety, depression or physical illness that can affect the patient's sleep quality. The amount of sleep changes with age. There is a decrease in the stages of sleep in old age, but some old individuals can adapt to physiological changes in maintaining sleep quality. [18].

Good sleep quality is determined by how a person prepares his sleep patterns at night such as falling asleep, the ability to stay asleep, and the ease of falling asleep 
without medical help. Good sleep quality is a basic human need.During sleep, there are various activities in the body that will affect physical and mental health. Respondents in this study mostly had poor sleep quality because they felt there was no other activity and did not feel worried about the illness they experienced and had become accustomed to the illness.

The dialysis vintage ( $\mathrm{p}$ Value 0.005 ) is a dominant factor which consists of shifts carried out hemodialysis and long hemodialysis. The duration of hemodialysis therapy can cause sleep patterns in patients with chronic renal failure. This occurs due to progressive symptoms and diseases from undergoing therapy or complications caused by long-term hemodialysis therapy or other sleep disorders such as increased parathyroid hormone, renal osteodystrophy, breathing disorders during sleep and excessive daytime.[19] [20] [21]

According to Sekercioglu, et al [22] [23] the first problem that needs to be addressed is the impact of dialysis on survival and sleep quality in hemodialysis patients. Based on characteristics at an early stage, this seems to be an important concern, because it will have a significant effect on quality of life._Hemodialysis therapy is associated with an $8 \%$ increase in the risk of death in ESRD. Similar results have been reported in patients with acute renal failure and chronic peritoneum. [24]

Laboratory factors in the data measured in this study are the causes of ESRD. This study shows that cholesterol levels are the most common cause of chronic kidney failure in respondents. High level of cholesterol is an increase in the level of lipids in the blood produced by the liver and controlled by blood levels. Increased high risk can reduce the body's ability to react to insulin or can stop insulin production altogether which can cause complications such as diabetes ketoacidosis, and longterm hyperglycemia that causes complications. Cholesterol is a high level of bad fat that is not normal and can have a negative impact on blood pressure. Bad cholesterol can also cause several complications, such as stroke, myocardial infarction, encephalopathy, and kidney failure. Any disease that causes pain and physical discomfort can affect sleep problems. Disease can force a person to sleep in an unusual position that can interfere with sleep [25] [26].

Using multivariate phased logistic regression analysis, we found that dialysis vintage was an independent predictor of poor sleep quality. This is consistent with many previous findings. Some studies have also found that poor sleep quality improves the inflammatory process in women with vintage dialysis more than 1 year compared to men, and increases the risk profile for high infectious events in women. [27][28][29][30]

\section{LIMITATION}

The significant relationship between risk factors and poor sleep quality was indicated by gradual logistic regression analysis. However, there are still some weaknesses in this study. First, the small numbers of samples and many other variants such as comorbidity and different dialysis modalities were not included for further analysis. In addition, our chosen participants were limited to rural areas in Central Java in Indonesia, the results of which may be far from hemodialysis patients in general. As a result, we need a large and long-term study in the future to confirm observations of other factors and results and to establish a stronger relationship between sleep disorders and related factors. Despite these limitations, this study attempts to highlight the high prevalence and negative effects of sleep quality, increasing awareness of the importance of recognizing and managing sleep disorders.

\section{CONCLUSIONS}

According to the results of this study, female hemodialysis patients need more attention in long-term care. In addition, it is necessary to pay attention to the possible relationship between poor sleep quality and laboratory analyzes that have been carried out on these patients, for example cholesterol levels so that immediate medical treatment can be done to reduce the accompanying symptoms. The duration of dialysis (vintage dialysis) that has been identified is often associated with poor quality of life. So, we also need to assess early and the treatment of sleep disorders for clinical outcomes remains important. It is recommended that patient management should improve sleep quality in addition to managing general medical problems.

\section{REFERENCES}

[1] et al Bailie GR, Larkina M, Goodkin DA, "Variation in intravenous iron use internationally and over time: the Dialysis Outcomes and Practice Patterns Study (DOPPS)," Nephrol. Dial. Transplant., no. 28(10), pp. 2570-2579.

[2] K. J. Kang SH, Do JY, Lee SY, "Effect of dialysis modality on frailty phenotype, disability, and health-related quality of life in maintenance dialysis patients," PLoS One, vol. 12(5), no. e0176814, 2017.

[3] P. Y. Pai MF, Hsu SP, Yang SY, Ho TI, Lai CF, "Sleep disturbance in chronic hemodialysis patients: the impact of depression and anemia," Ren Fail, vol. 29(6), no. 673-677, 2007.

[4] et al Eryavuz N, Yuksel S, Acarturk G, "Comparison of sleep quality between hemodialysis and peritoneal dialysis patients," Int Urol Nephrol, vol. 40(3), no. 785-791, 2008.

[5] W. E. Weiss M, Mettang T, Tschulena U, "Health-related quality of life in haemodialysis patients suffering from chronic itch: results from GEHIS (German Epidemiology Haemodialysis Itch Study)," Qual Life Res, vol. 25(12), no. 3097-3106.

[6] et al Merlino G, Piani A, Dolso P, "Sleep disorders in patients with end-stage renal disease undergoing dialysis therapy. Nephrology, dialysis, transplantation : official publication of the European Dialysis and Transplant Association," Eur. Ren. Assoc., vol. 21(1), no. 184-190.

[7] Anand S, "Prevalence of chronic kidney disease in two major Indian cities and projections for associated cardiovascular disease," Int. Soc. Nephrol., 2015.

[8] H. H. Iseki K, Tsuruya K, Kanda E, Nomura T, "Effects of sleepiness on survival in Japanese hemodialysis patients," $J$ DOPPS study. Nephron Clin Pr., vol. 128(3-4), pp. 333-340, 2014. 
[21] et al Tomita T, Yasui-Furukori N, Oka M, "Insomnia in patients on hemodialysis for a short versus long duration," Neuropsychiatr Dis Treat, vol. 12, no. 2293-2298, 2016. Diagn Res, vol. 10(12), no. Lc01-1c04.

[10] et al Hasheminasab Zaware R, Mahmoodi Meymand MH, Rezaeian M, "Insomnia and Restless Leg Syndrome in Patients Undergoing

Chronic Hemodialysis in Rafsanjan Ali Ibn Abitaleb Hospital.,"

Nephrourol Mon, vol. 8(1), no. e29527.

[11] Alim IZ, Uji validitas dan reliabilitas instrumen pittsburgh sleep quality index versi Bahasa Indonesia $=$ Test validity and reliability of the instrument pittsburgh sleep quality index Indonesia language version. 2015.

[12] et al Giannaki CD, Sakkas GK, Karatzaferi C, "Evidence of increased muscle atrophy and impaired quality of life parameters in patients with uremic restless legs syndrome," PLoS One, vol. 6(10), no. e25180, 2011.

[13] B. G. Jaracz K, Kalfoss M, Gorna K, "Quality of life in Polish respondents: psychometric properties of the Polish WHOQOLBref," Scand. J. Caring Sci., vol. 251-260, no. 20(3).

[14] et al Han M, Williams S, Mendoza M, "Quantifying Physical Activity Levels and Sleep in Hemodialysis Patients Using a Commercially Available Activity Tracker.," Blood Purif, vol. 41(1-3), no. 194-204, 2016.

[15] A. S. Sabet R, Naghizadeh MM, "Quality of sleep in dialysis patients," Iran J Nurs Midwifery Res, vol. 17(4), no. 270-274.

[16] R. Y. Zhou H, Li W, "Poor sleep quality of third trimester exacerbates the risk of experiencing postnatal depression," Psychol Heal. Med, no. 1-10.

[17] M. J. Kosmadakis GC, "Sleep disorders in dialysis patients. Int J Artif Organs," vol. 31(11):, no. 919-927.

[18] et al Lai X, Chen W, Bian X, "Predictors of poor sleep quality and excessive daytime sleepiness in peritoneal dialysis patients. .:.," Ren Fail, vol. 37(1), no. 61-65.

[19] A.-J. H, “A comparison of sleep disturbances and sleep apnea in patients on hemodialysis and chronic peritoneal dialysis," Saudi $J$ Kidney Dis Transpl, vol. 22(5), no. 922-930.
[22] B. B. Sekercioglu N, Curtis B, Murphy S, "Sleep quality and its correlates in patients with chronic kidney disease: a crosssectional design," Ren Fail, vol. 37(5), no. 757-762.

[23] U. M. Novak M, Winkelman JW, "Restless Legs Syndrome in Patients With Chronic Kidney Disease," Semin Nephrol, vol. 35(4), no. 347-358.

[24] A. M. Noshad H, Sadreddini S, Nezami N, Salekzamani Y, "Comparison of outcome and quality of life: haemodialysis versus peritoneal dialysis patients," ingapore Med J, vol. 50(2), no. $185-192$

[25] F. J. Tarp H, Bonde-Petersen M, "Patients in Haemodialysis Experienced Uraemic Pruritus as a Dual Phenomenon," J Ren Care, vol. 43(1), no. 21-28.

[26] D. A. Tuncel D, Orhan FO, Sayarlioglu H, Isik IO, Utku U, "Restless legs syndrome in hemodialysis patients: association with depression and quality of life," Sleep Breath, vol. 15(3), no. 311-315.

[27] H. F. Saeedi M, Shamsikhani S, Varvani Farahani P, "Sleep hygiene training program for patients on hemodialysis," Iran $J$ Kidney Dis, vol. 8(1), no. 65-69.

[28] B. F. Scherer JS, Combs SA, "Sleep Disorders, Restless Legs Syndrome, and Uremic Pruritus: Diagnosis and Treatment of Common Symptoms in Dialysis Patients," Am J Kidney Dis, vol. 69(1), no. 117-128.

[29] et al Wang SY, Zang XY, Fu SH, "Factors related to fatigue in Chinese patients with end-stage renal disease receiving maintenance hemodialysis: a multi-center cross-sectional study. .:.,"Ren Fail, vol. 38(3), no. 442-450, 2016.

[30] et al Yeganeh B, Rezaei Moghadam A, Alizadeh J, "Hepatitis B and $\mathrm{C}$ virus-induced hepatitis: Apoptosis, autophagy, and unfolded protein response," World J Gastroenterol, vol. 21(47), no. $13225-13239$. et al Roumelioti ME, Argyropoulos C, Pankratz VS, . "Objective and subjective sleep disorders in automated peritoneal dialysis," Can J Kidney Heal. Dis, no. 3:6, 2016. 